\title{
Commentary on "A Nationwide Survey of Knowledge of and Compliance with Cancer Pain Management Guidelines by Korean Physicians"
}

\author{
Kieran Walsh, FRCPI \\ BMJ Learning, BMJ Group, BMA House, London, UK
}

Kim et al. [1] have presented the results of a fascinating study that seems to show that "better knowledge of cancer pain management resulted in greater reduction of patient pain severity."

However it is a moot point as to whether some physicians genuinely did have improved knowledge or indeed whether patient pain severity was reduced in certain patients. The method used to assess the knowledge of physicians was by means of true/false multiple choice questions. However this form of assessment has increasingly been discarded by postgraduate examining authorities as it lacks reproducibility and validity. Single best answer and extended matching questions have now largely taken over as the assessment format of choice.

Also the reduction of patient pain severity was judged by retrospective case notes review-however some patients' pain may not have been recorded in the case notes. Case notes are simply not always a reliable means of judging the effectiveness of clinical care.

Putting these caveats aside, there may well be a relationship between physician knowledge of pain management and reduced patient pain severity in patients. If that is the case, then a strategic approach should be undertaken to improve knowledge levels. However good applied knowledge is just one of the components necessary to achieve better care. Good problem solving and procedural skills as well as healthy attitudes and improved behaviours are also necessary. Medical education interventions to improve care will need to tackle all these areas.

\section{Conflicts of Interest}

Conflict of interest relevant to this article was not reported.

\section{Reference}

1. Kim DY, Ahn JS, Lee KH, Kim YC, Lee J, Kim SY. A nationwide survey of knowledge of and compliance with cancer pain management guidelines by Korean physicians. Cancer Res Treat. 2014;46:131-40.

Correspondence: Kieran Walsh, FRCPI

BMJ Learning, BMJ Group, BMA House, Tavistock Square, London WC1H 9JR, UK

Tel: 44-7985-755-333, Fax: 44-207-383-6242, E-mail: kmwalsh@bmjgroup.com

Received May 26, 2014 Accepted July 16, 2014 